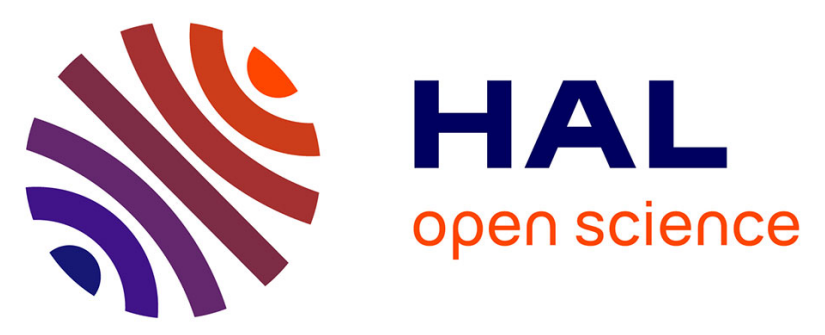

\title{
Decision Problem of Instrumentation in a Company involved in ISO 50001
}

Bastien Rizzon, Vincent Clivillé, Sylvie Galichet, Pascal Ochalek, Elodie Ratajczak

\section{- To cite this version:}

Bastien Rizzon, Vincent Clivillé, Sylvie Galichet, Pascal Ochalek, Elodie Ratajczak. Decision Problem of Instrumentation in a Company involved in ISO 50001. IESM International Conference on Industrial Engineering and Systems Management, October 2015, Seville, Spain, Escuela Superior de Ingenieros - Universidad de Sevilla, Oct 2015, Sevilla, Spain. 8 p. USB support. hal-01224230

\section{HAL Id: hal-01224230 \\ https://hal.science/hal-01224230}

Submitted on 4 Nov 2015

HAL is a multi-disciplinary open access archive for the deposit and dissemination of scientific research documents, whether they are published or not. The documents may come from teaching and research institutions in France or abroad, or from public or private research centers.
L'archive ouverte pluridisciplinaire HAL, est destinée au dépôt et à la diffusion de documents scientifiques de niveau recherche, publiés ou non, émanant des établissements d'enseignement et de recherche français ou étrangers, des laboratoires publics ou privés. 


\title{
Decision Problem of Instrumentation in a Company involved in ISO 50001
}

\author{
(presented at the $6^{\text {th }}$ IESM Conference, October 2015, Seville, Spain) (C) $\mathrm{I}^{4} \mathrm{e}^{2} 2015$
}

\author{
Bastien Rizzon \\ LISTIC- Université Savoie Mont Blanc \\ Email: bastien.rizzon@univ-smb.fr
}

\author{
Vincent Clivillé \\ Sylvie Galichet \\ LISTIC- Université Savoie Mont Blanc \\ Email: vincent.cliville@univ-smb.fr \\ Email: sylvie.galichet@univ-smb.fr
}

\author{
Pascal Ochalek \\ Elodie Ratajczak \\ Adixen by Pfeiffer Vaccum \\ Email: pascal.ochalek@adixen.fr \\ Email: elodie.ratajczak@adixen.fr
}

\begin{abstract}
In the context of performance management and the involvement of companies in sustainable development, energy management is a new challenge. Managing energy is a complex problem as it can potentially impact the whole company and if mishandled could be harmful for its added value. In order to assist companies in this challenge, the ISO 50001 proposes a standard for the creation of Energy Management Systems (EnMS). Such systems bring the recurring problem of decision about the choice of the actions necessary into their deployments. Notably, they rely heavily on the data related to the consumptions of energy and its usage in a given company. Hence the problem of decision, at an audit stage, can concern the choice of a sound instrumentation. This paper deals with the interest of the company adixen Vacuum Products in a standard for decision aiding in this situation. The idea is to build the model of preference of the Decision Maker and to establish a generic procedure about decision supported by a MCDA tool. Our proposition is to use the ACUTA method in order to elicit this model of preference.
\end{abstract}

\section{INTRODUCTION}

At the start of the 21st century, energy savings and the limitation of greenhouse gas emissions have become major challenges for many organizations including companies. Thus the International Standard Organization (ISO) estimates the potential gain to be about $30 \%$ on the energy consumption. In this context the ISO edited a specific norm for the companies involved in the control of their consumptions of energy through a system of management: the ISO 50001, published in 2011 [1] .

The expectations and the major role of instrumentation for such a system of management are now presented.

\section{A. The ISO 50001 concerning the Instrumentation}

The ISO 50001, Energy Management Systems Requirements with guidance for use, gives organizations the requirements for Energy Management Systems (EnMS) as a standard. It provides a framework of requirements enabling organizations to:

- Develop a policy for more efficient use of energy

- Fix targets and objectives to meet the policy

- Use data to better understand and make decisions concerning energy use and consumption
- Measure the results

- Review the effectiveness of the policy

- Continually improve energy management.

According to [2] "ISO 50001 includes requirements for a program of energy data collection such as the installation of Automatic Monitoring and Targeting (AMT) systems, and this can be used to increase energy efficiency at different levels of a factory". This can be seen as the application for an EnMS of the well known principle of "What you measure is what you get" [3] [4].

Usually, a company has knowledge about one point of measurement for a given energy: a single global point available directly with a counter or indirectly through the related bills. Intuition or practical observations point to the lack of information in such a single point of measurement. There is also the possibility of taking the reading directly on an equipment. This is generally considered as a timeconsuming activity with many possibilities for errors. However the exhaustive instrumentation of equipments and machines in order to measure their consumptions of energy is expensive, time consuming, detrimental for the company's activities of production or $R \& D, \ldots$ For example such an instrumentation in order to get the electrical consumption in real time of a single equipment can cost from $600 €$ to $1500 €$. So, a company which cannot afford to "measure everything" has to plan for the instrumentation of its activity in order to select the most relevant measurement points.

Before presenting the instrumentation decision problem, let us recall some key points about the general framework of improvement in companies, the so called Quality Management System (QMS) which is very similar to the EnMS. Indeed according to [1], the ISO 50001 is built with the same common elements as the other ISO standards, including the ISO 9001 which deals with QMS and is well known by many companies.

\section{B. Quality Management System}

According to the ISO 9001, the QMS is based on the PDCA cycle often illustrated by the Deming's wheel [5]. More precisely, improvement must be made Step by Step, must be related to the company's processes according to its Process 
Mapping. Every action must be controlled before launching the next step. In this way the PDCA four stages are itemized as follows:

- Plan: the establishment of the objectives and the development of the action plan,

- Do: the deployment of the action plan,

- Check: the measurement of the results with regard to the planned objectives,

- Act: the corrections and improvements of the plan and its deployment (before the next cycle).

We call Step one cycle of the Deming's wheel. These steps are usually marked with the adoption of standards in order to capitalize on the experience gained in the process of Continuous Improvement. In order to control the successive steps of the improvement, Process Managers need decision aiding.

In this study, decision aiding concerns the instrumentation of equipments in order to provide energy consumptions related to process and activities to their managers. The proposed idea is to provide a decision aiding framework easily usable for all the steps of the improvement. Let us detail this problem of decision in the next subsection.

\section{A Decision Problem for Instrumentation}

The decision process has to establish a balance with:

- the connection between the electrical network and the Process Management in the company,

- the degree of accuracy in the energy consumption mapping,

- the costs in time, resources,... allocated to the instrumentation.

The company is interested in standardizing this process in order to replicate it for other perimeters of its activity. During this process of adopting the standards of the ISO 50001 that deal with energy management (which impacts all the company's activities) the rigorous and systematized process is also defined. In order to deal with this problem, this paper is based on the case presently studied in the company adixen Vacuum Products (aVP). The idea is to build with $\mathrm{MCDA}^{1}$ approaches of the MAUT ${ }^{2}$ family [6] the model of preference of the DM for the problem of decision concerning instrumentation. More precisely a method based on preference disaggregation for their holistic characteristics is adopted. Amongst these methods, ACUTA [7] is appreciated by the company for its interactivity.

This paper is organized around two main sections. Section II presents aVP and its situation with regard to energy management. It also shows how the deployed instrumentation for a part of the electrical network of the company has been matured since it had an influence in the decision making

\footnotetext{
${ }^{1}$ MultiCriteria Decision Analysis

${ }^{2}$ Multiple Attribute Utility Theory
}

process. Section III presents the deployment a posteriori of a MCDA method, namely ACUTA, in order to build the model of preference of the DM for this particular question of instrumentation. Then thus model could be used for the next step(s) of improvement.

\section{Preliminary AnAlysis of EnERgy Consumptions}

\section{A. Presentation of the Company aVP}

Since 2011, aVP has been a French subsidiary of the Pfeiffer Vacuum Technology AG Group. The group's primary activity is to offer solutions based on vacuum technology for a broad range of applications, including the semi-conductor, analytic instrument, coating and R\&D markets, as well as a variety of general manufacturing sectors. These applications are represented in aVP's sectors of activity, notably:

- $\quad$ productions,

- $\quad$ supports to production,

- R\&D,

- $\quad$ services.

These sectors of activity rely on a large know-how in the field of mechanics and electronics and the infrastructure related built from the 1950's to the present days. Certified ISO 9001 and 14001, aVP is now aiming for the certification ISO 50001 in alignment with its management organization. In order to support the management of its sectors of activity, the company has purchased and installed a software infrastructure that allows to build custom, Web-enabled applications for accessing, automating and controlling "smart" devices in real-time over the Internet.

Now that the activity of the company is described from a management point of view, the next subsection will focus on the energy consumed, starting from a financial point of view.

\section{B. Existing Data about Energy Consumptions}

The company is supplied with two types of energy:

- $\quad$ Electricity

- Gas

It is important to note that there is no interaction between these two types of energy. Indeed for aVP gas is only involved for comfort heating and electricity is not involved in heating in the company. So the two types can be discriminated in the EnMS without the risk of missing a potential interaction. A fast analytical work based on the bills related to these energies has shown that about $90 \%$ of the total of the energy costs are related to electricity. Thanks to this information the DM has been able to argue for an approach that should focus on electricity initially.

However the single global value of electric consumption emitted every month on the bill is not sufficient in terms of specification (considering the different sectors of activity, buildings,... in the company). So it is relevant to "go beyond" the global point of view. 


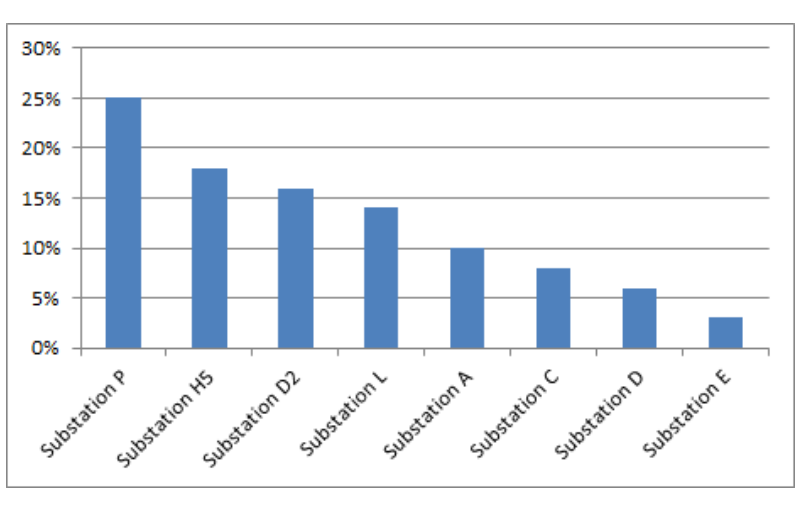

Fig. 1. Electric Substations Relative Consumptions

The global electrical station distributes electricity to 8 substations. Each substation is already instrumented with electrical counters that allow to record the consumptions related to each substation. A fast analytical work based on these data has shown the distribution of electricity amongst the 8 substations (see figure 1). According to this result the substation $\mathrm{P}$ is the major point of consumption.

The next stage now is to check what is beyond the substation $\mathrm{P}$ and to figure if actions can be decided from this point of view. To achieve this we proceeded to specify the equipments related to the substation and the existing readings.

It has appeared quickly that it was impossible to follow the electricity consumption of an equipment and its related sector of activity from the readings of the substation. Substation $\mathrm{P}$ distributes electricity for equipments in areas that were progressively built during the last decades. So this electrical network distributes electricity for machining, assembly lines, services, design and research,... So it is very difficult to match the energy consumptions with the Process Mapping which is mandatory for the EnMS because the future energy consumption gains will be managed by the Process Managers. Furthermore there is no instrumentation existing in the equipments related to substation $\mathrm{P}$.

For the EnMS the DM wants to develop something close to the Management by Process. However the diverse sectors of activity and so their management are mixed in the substation $P$ counter. In order to decide on the adequate instrumentation the first thing was to map the network of equipments related to substation $\mathrm{P}$ with the input by expertise of the equipments known as consumptive and their related sector of activity.

\section{Instrumentation for the Electric Substation $P$}

Figure 2 is the illustration of the Electrical Network of Substation $\mathrm{P}$ represented as an organizational tree:

- The root of the tree (level 1) is the electrical cabinet which distributes electricity to the many elements of substation $\mathrm{P}$.

- The leaves of the tree (level 2 to 5) are the equipments or machines supplied by the substation $\mathrm{P}$.

- The electrical consumption read on a Parent Node is the direct sum (plus non-insignificant loss) of the consumptions of its Children Node (with the exception of one generator illustrated by its own symbol in the tree).

The nodes are considered as points of interest for the EnMS which can all be instrumented. The nodes are categorized depending of the sector of activity concerned with the equipment. When all the children of a node belong to the same sectors of activity, the parent node is categorized the same way. When the children of a node are of different sectors of activity, the parent node is considered as a Mix node.

The nodes noted as important consumers were identified by expertise. The other nodes are either non important consumers or the expert could not assess them.

Supported by this new information the DM decided for an instrumentation (see section III-A6). He/she conducted meetings and brainstorming in order to take into consideration the many constraints in the project of instrumentation. The problem was handled without the support of a MCDA tool.

The idea presented in the next section is to build the model of preference that would have been elicited if a MCDA approach had been deployed. By doing so, the next step(s) in the process of Continuous Improvement (the instrumentation of substation $\mathrm{H} 5$ for instance) would thus in this way benefit from this model in its decision process a priori.

\section{Elicitation of the Model of Preference}

In this section, the ACUTA method will be deployed on the practical case of the instrumentation of the most consumptive electric substation in the company.

The method is run a posteriori of the decision for this instrumentation. However the process here is to rebuild the considerations of the DM when he/she decided for a specific instrumentation into a Multicriteria Decision Problem. By deploying ACUTA on this problem the DM would get information about the rigor behind the decision and to improve the process of such a decision for the next action plans. In this paper the rigor to be expressed is the balance operated between the different criteria considered in his/her process of thought for deciding which alternative to implement for the company's EnMS. This balance will be expressed in the model of preference.

The different alternatives and criteria in this decision problem will now be detailed with their motivations.

\section{A. Description of the Alternatives}

1) Initial Instrumentation (a01): This is the state of the instrumentation before the deployment of the new instruments. This is considered as an alternative for the DM who could chose to Do nothing if the other alternatives were not worth the effort. Furthermore it is representative of a "global" measurement framework.

This instrumentation consists of a single point for measurement at the root of the electrical network (see figure 2). 


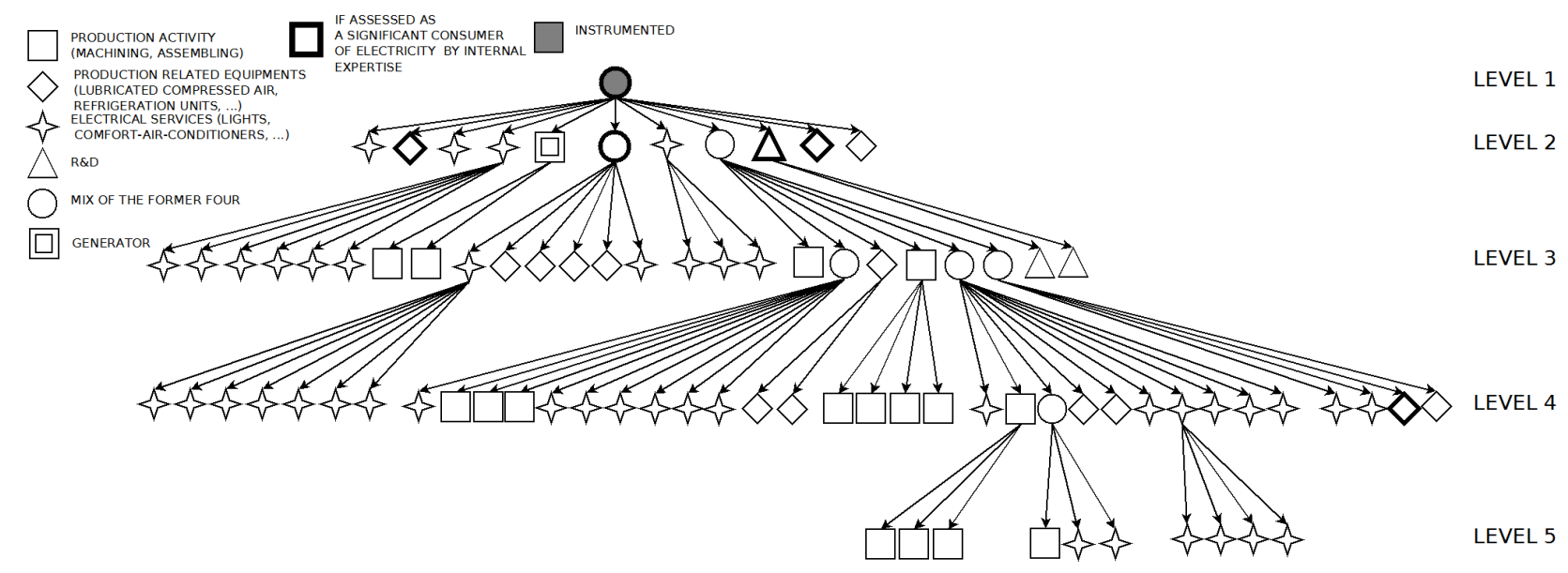

Fig. 2. Electrical Network Illustration

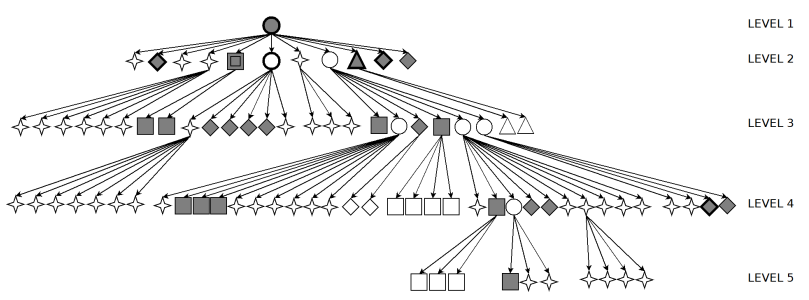

Fig. 3. Illustration for Instrumentation a04

2) Exhaustive Instrumentation (a02): The idea of this alternative is to provide information related to electrical needs in the most accurate way by monitoring every final element of electrical consumption in the network.

By adding these values, according to the structure of the network, every node can be controlled.

This instrumentation consists of the deployment of points of measurement for every leaf in the network (see figure 2).

3) Sub-global Instrumentation (a03): The idea of this alternative is to provide information related to electrical needs for the level in the network just below the global point of view.

This alternative is considered by the DM who wants to assess if going "one step further" of the global point of view would be satisfactory.

This instrumentation consists of the deployment of points of measurement for every node in level 2 of the network (see figure 2).

4) Sectors of Activity Instrumentation (a04): The idea of this alternative is to provide information related to electrical needs that can be linked to the different sectors of activity in the company.

This alternative is considered by the DM because it would be easier to make the link between a dysfunction in electrical consumption and the related sector of activity.

This instrumentation consists of the deployment of points

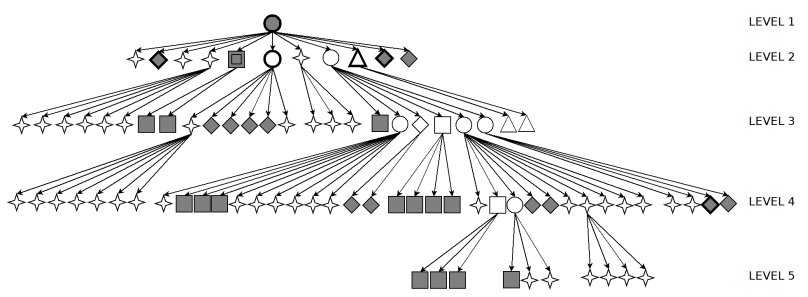

Fig. 4. Illustration for Instrumentation a05

of measurement, according to the structure of the network, in a way that allows to discriminate measurements as belonging to a given sector of activity. It is illustrated by figure 3 with grayed nodes as instrumented nodes.

In practice: if a parent node can be classified in another category than MIX it is to be instrumented; if not check for the children nodes and so on.

5) Production Centered Instrumentation (a05): The idea of this alternative is to provide information related to electrical needs for the added value activity of the company.

This alternative is considered by the DM because it highlights a critical aspect of the strategy of the company: Balancing its energetic needs with the quality of its lucrative activity.

This instrumentation consists of the deployment of point of measurements on the node related to the Production activity of the company, trying as much as possible to be accurate and is illustrated by figure 4 .

In practice: every leaf node belonging to the Production or Production Related categories are to be instrumented (represented by a grayed node).

6) Handpicked Instrumentation (a06): The idea of this alternative is to provide information related to electrical needs for a set of nodes that the DM chose one by one.

This alternative is considered by the DM who wants to consider a non-systematic approach for the instrumentation while focusing on the most consumptive equipments (identified 


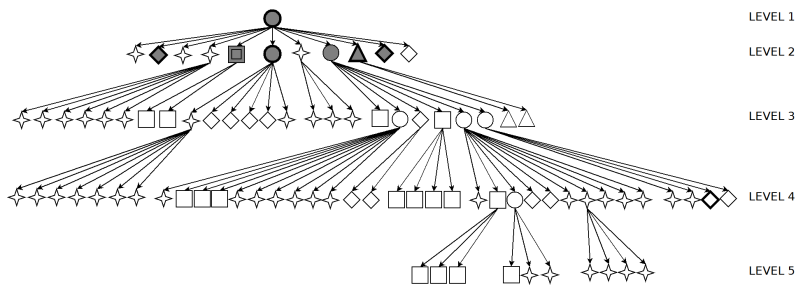

Fig. 5. Illustration for Instrumentation a06

by expertise).

This instrumentation consists of the deployment of points of measurements on the node selected for reasons specific to the company's needs. It can be the result of a process of selection during a brainstorm and is illustrated by figure 5 with grayed nodes as instrumented nodes.

This is the list of the alternatives in the problem, now let us see about the criteria.

\section{B. Description of the Criteria}

1) Compatibility with the Governing Organization of the Company ( $(1)$ : This criterion allows for the assessment of the concerned alternative according to the ability to give information about electrical consumptions directly to the manager concerned.

The criterion is defined from $\alpha_{1}=0$ to $\beta_{1}=10$ as a scale. In order to build this scale, the DM must verify that the score increases progressively according to his/her satisfaction. The higher the value is, the closer to the governing organization of the company the instrumentation is.

This is a Gain criterion as the DM is more satisfied when the instrumentation allows the breakdown of electrical consumptions into something similar to the governing organization of the company.

2) Total Distance between the Points of Measurements ( $g 2)$ : This criterion allows the assessment of the concerned alternative according to a technical constraint. In this case study, the technical constraint is about the cabling of the different points of measurements for the alternatives concerned.

The criterion is defined from $\alpha_{2}=0 \mathrm{~m}$ to $\beta_{2}=200 \mathrm{~m}$. Every $100 \mathrm{~m}$ a repeater has to be installed in the cabling. Furthermore the disturbance for the activity of the company is higher when the action of instrumentation concerns a bigger area. The DM does not want more than 1 repeater to be installed, so the upper limit for this criterion is $200 \mathrm{~m}$.

This is a Cost criterion as the DM is more satisfied when the installations concerned are closer to each other.

3) Durability of the Instrumentation (g3): This criterion allows the assessment of the alternative concerned according to the longevity of the physical equipments to be monitored. The machine can be replaced or displaced which is detrimental for the DM because it would be necessary to reconfigure the cabling after such an important modification.

This criterion is defined from $\alpha_{3}=0$ months to $\beta_{3}=24$ months. 0 means that the equipment or the machine is to move "today" while 24 means that the equipment or the machine is not to move before 24 months at least. This is the upper limit for this criterion as such action plans are seldom defined beyond a 2 year horizon.

This is a Gain criterion as the more durable the equipment or machine will be, the more satisfied the DM is.

4) Expectation in a Gain for the Energy Management System (g4): This criterion allows the assessment of the alternative concerned according to the expected outcome in order for the EnMS to be built. Data about this criterion are uncertain because the evaluation of the future gains is a costing and difficult task. So in fine this criterion remains "qualitative".

The criterion is defined with a reasonable linguistic scale of 3 terms: low, medium, high. For the sake of simplicity in this case study these linguistic values are turned into the numeric values $1,2,3$, i.e $\alpha_{4}=1$ and $\beta_{4}=3$.

This is of course a Gain criterion.

5) Total Cost (g5): This criterion allows the assessment of the concerned alternative according to the total cost of the instrumentation which is self explicit.

The criterion is defined according to a fixed price of 700 $€$ per point of measurement. If the DM is constrained by a budget limit some alternatives could be immediately discarded through this criterion.

This is of course a Cost criterion ranging from $\alpha_{5}=0 €$ to $\beta_{5}=47600 €$

Now that we have described the alternatives and the criteria, the next subsection will deal with the different inputs required for running ACUTA with the Diviz software [8].

\section{Inputs}

Figure 6 is an illustration of the input for using the ACUTA method through Diviz of the Decision Deck [8].

The inputs named alternatives and criteria are simply the identification of the alternatives (here $a_{01}$ to $a_{06}$ ) and the criteria (here $g_{1}$ to $g_{5}$ ).

The input named preference Direction is about the nature of each criterion as a Gain or Cost criterion.

All these inputs were already figured in the III-A and III-B. Let us see about the others.

1) performanceTable: This is the Decision Matrix (see table I) where the values describe each alternative from the point of view of the 5 criteria of the problem. These values are obtained from estimations, measurements, linguistic expressions,... 


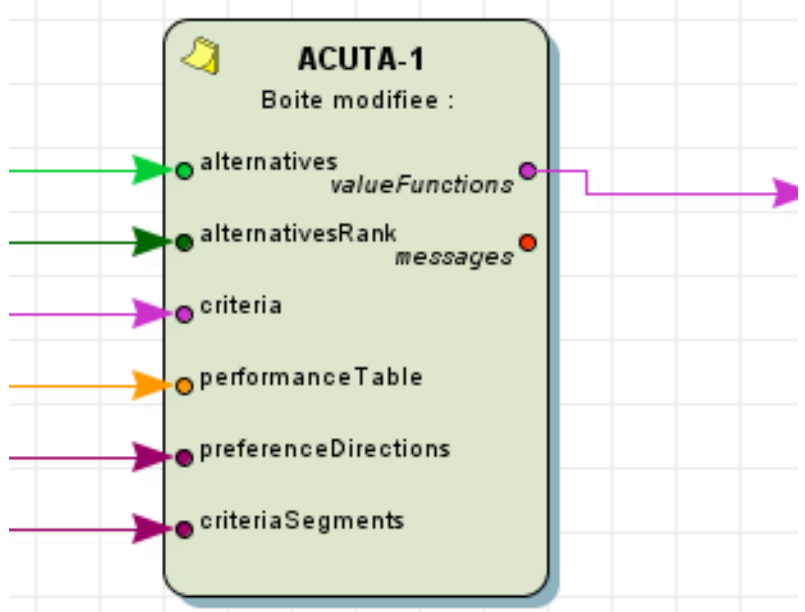

Fig. 6. The Toolbox ACUTA on Diviz

TABLE I. THE DECISION MATRIX FOR THIS PROBLEM OF INSTRUMENTATION

\begin{tabular}{|c|c|c|c|c|c|}
\hline & g1 & g2 & g3 & g4 & g5 \\
\hline a01 & 0 & 0 & 24 & Low & 0 \\
\hline a02 & 10 & 200 & 6 & Medium & 47600 \\
\hline a03 & 7 & 10 & 20 & Medium & 7700 \\
\hline a04 & 9 & 120 & 12 & High & 16100 \\
\hline a05 & 8 & 80 & 6 & High & 19600 \\
\hline a06 & 6 & 5 & 20 & Medium & 4200 \\
\hline
\end{tabular}

2) alternativesRank: This is the order of reference established by the DM. The DM expressed his/her preference directly over the actions, such as for example "I prefer each other alternative over the exhaustive instrumentation; I prefer the level 2 instrumentation over initial instrumentation" and so on. In the end, the preferences of the DM were expressed with the following reference order:

$$
a_{06} \succ a_{05} \succ a_{04} \succ a_{03} \succ a_{01} \succ a_{02}
$$

3) criteriaSegments: This input concerns the number of segments for each value function. In this case study, the number has been set to 2 for all criteria.

So the model of preference will take the form of five 2piece linear value functions as $g_{i}\left(a_{k}\right) \mapsto u_{i}\left(g_{i}\left(a_{k}\right)\right)$ with their breakpoints at $\gamma_{i}$ such as $\gamma_{i}=\frac{\alpha_{i}+\beta_{i}}{2}$.

The ACUTA method can now be run in order to compute a model of preference. It is important to note that the model of preference is not unique, but ACUTA elicits a single model of preference by trying to respect at best all the constraints of the problem at once (see [7] for more details). There are other approaches that deal with the elicitation of a single "representative" model of preference with preference disaggregation methods (see [9]). A considered benefit for the choice of ACUTA is the location of the solution, "as far as possible" from the boundary of the constraints polyhedron.

\section{Output: valueFunctions}

This is the model of preference of the DM for this problem of instrumentation. This model is synthesized in table II and
TABLE II. The Model of Preference Computed with ACUTA

\begin{tabular}{|c||c|c||c|c||c|c|}
\hline$g_{i}$ & $\alpha_{i}$ & $u_{i}\left(g_{i}\left(\alpha_{i}\right)\right)$ & $\gamma_{i}$ & $u_{i}\left(g_{i}\left(\gamma_{i}\right)\right)$ & $\beta_{i}$ & $u_{i}\left(g_{i}\left(\beta_{i}\right)\right)$ \\
\hline$g_{1}$ & 0 & 0 & 5 & $\mathbf{0 , 1 0}$ & 10 & $\mathbf{0 , 1 3}$ \\
\hline$g_{2}$ & 0 & $\mathbf{0 , 2 5}$ & 100 & $\mathbf{0 , 1 3}$ & 200 & 0 \\
\hline$g_{3}$ & 0 & 0 & 12 & $\mathbf{0 , 0 3}$ & 24 & $\mathbf{0 , 0 8}$ \\
\hline$g_{4}$ & 1 & 0 & 2 & $\mathbf{0 , 1 0}$ & 3 & $\mathbf{0 , 3 2}$ \\
\hline$g_{5}$ & 0 & $\mathbf{0 , 2 3}$ & 23800 & $\mathbf{0 , 0 7}$ & 47600 & 0 \\
\hline
\end{tabular}

TABLE III. Utility of THE Alternatives COMPUTED WITH ACUTA-DIVIZ

\begin{tabular}{|c||c|}
\hline Alternatives & Utilities \\
\hline $\mathrm{a} 01$ & 0,55 \\
\hline $\mathrm{a} 02$ & 0,25 \\
\hline $\mathrm{a} 03$ & 0,69 \\
\hline $\mathrm{a} 04$ & 0,7 \\
\hline $\mathrm{a} 05$ & 0,71 \\
\hline $\mathrm{a} 06$ & 0,71 \\
\hline
\end{tabular}

illustrated in figure 7.

The corresponding utility given to the alternatives can be seen in table III.

The DM can now figure the relative importance he/she had in mind for the different criteria according to the Decision Matrix and his/her preferences. From this model of preference the utility of an alternative given its evaluation according to the five considered criteria can be calculated.

\section{E. Discussing the Model of Preference}

Hence it is possible to discuss with the DM about the validity of the model of preference. The DM could either keep the model of preference as it is, in order to see if it can be considered as robust before testing it in the next instrumentation. Or the DM could disagree with the model of preference according to his/her judgment and so it would provoke questions about the Decision Matrix, the enrichment of the information given on the DM's preferences, changing the number of pieces in the value functions, ...

Assuming that this model is reliable, it could now be used to assess different or future alternatives in comparison to each other. Let us consider the alternative Energy Consumptive Equipments Instrumentation $a_{07}$. This instrumentation consists of the instrumentation of the equipments judged by expertise as consumptive. The evaluation of the alternative for each criterion is as follows:

$$
\begin{gathered}
g_{1}\left(a_{07}\right)=7 ; g_{2}\left(a_{07}\right)=100 ; g_{3}\left(a_{07}\right)=20 ; \\
g_{4}\left(a_{07}\right)=2 ; g_{5}\left(a_{07}\right)=4200
\end{gathered}
$$

With the model of preference the utility for this alternative is 0,61 , and so it is preferred only to $a_{02}$ and $a_{01}$. But the model of preference itself is not the only gain in this approach.

\section{F. Acquired Experience}

The whole approach deployed for this problem of instrumentation can now be standardized for the next step in Continuous Improvement. This standardization at a strategic level is illustrated by the figure 8 . 
g1
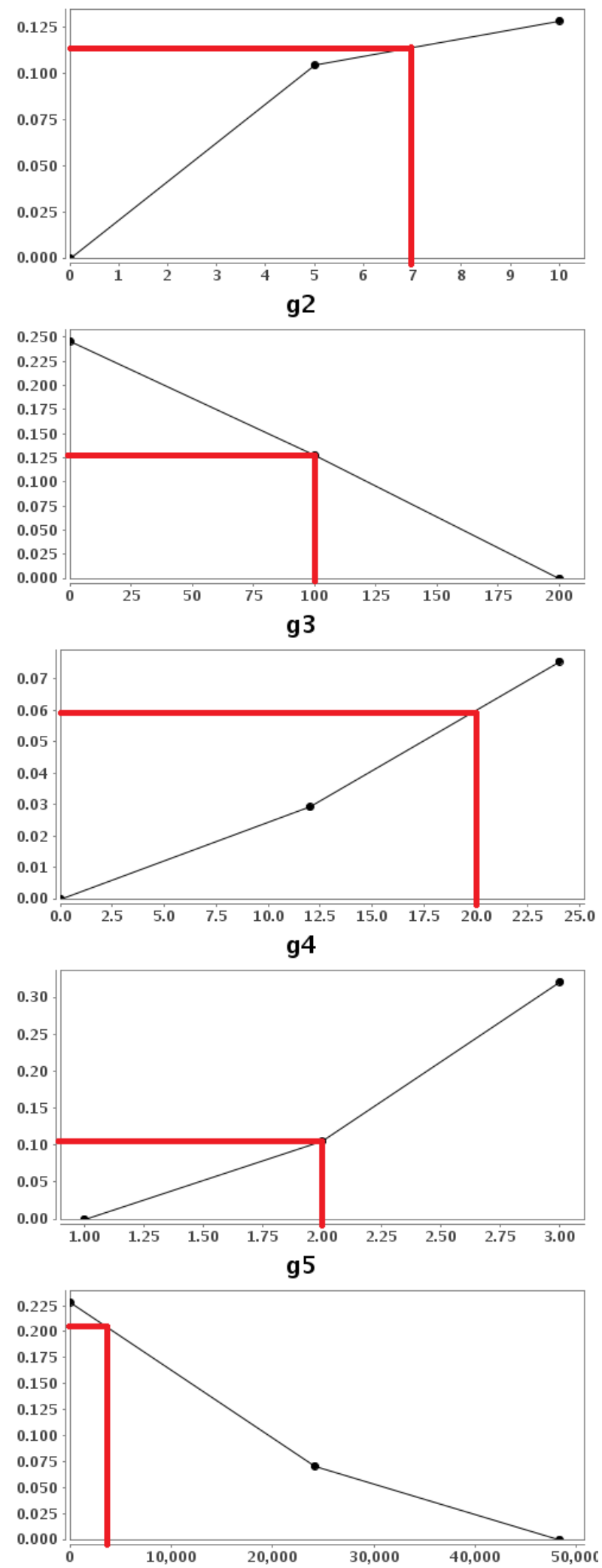

Fig. 7. Plot of the Model of Preference

Furthermore from the making of the problem of decision concerning instrumentation to the elicitation and interaction

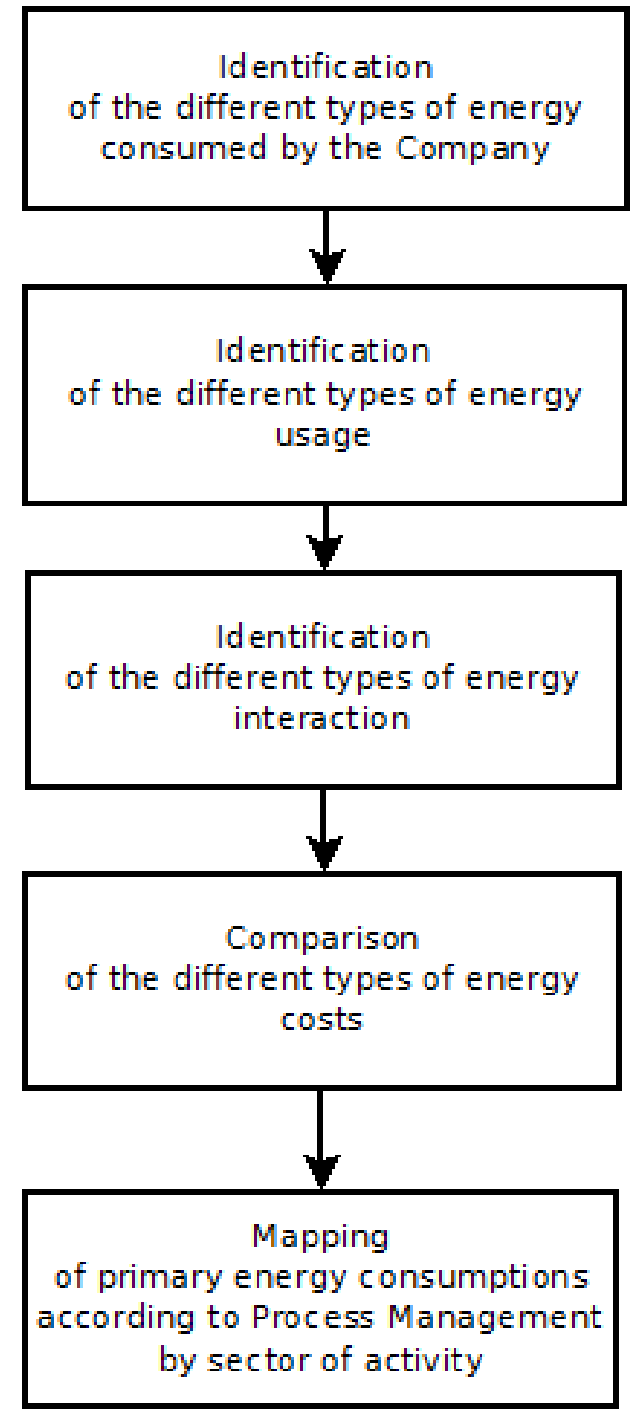

Fig. 8. Adopted Standardized Strategic Approach for Energy Monitoring in aVP

with the DM about the model of preference, the company has acquired some knowledge it was not directly seeking in the instrumentation problem. For example the Electrical Network scheme from a management point of view is now a document of support for mapping the company's activity more accurately. In order for the criterion $g_{4}$ to be correctly assessed we carried out information processing on existing data related to energy consumptions of aVP. This has allowed to identify a dysfunctional equipment and to understand how it should be dealt with. This experience has helped to refine the framework that the DM wants for the EnMS.

\section{DISCUSSION}

We proposed in this paper a practical approach for aiding a DM to obtain a more rigorous point of view on his/her own decision. The case studied here dealt with the required instrumentation to build an EnMS as a sound management system according to the related official standards. In order to do so, a multicriteria model of preference has been elicited by interacting with the DM and the company's existing data. 
This model is still to be refined and improved. For instance the preponderant criterion $g_{4}$ could be turned into a "quantitative" criterion when the gain for the EnMS can be measured and analyzed after the complete implementation of the instrumentation. Despite the potential weakness of the model of preference in its present state, the fact of building it from the making of the problem of decision is considered to be rich in information and experience. The approach has been adopted as a standard by aVP and after discussion is to be generalized for the other substations or adapted for other problems of decision concerning management systems.

However this standard was developed in a favorable situation with no interaction between the types of energy used by the company. Furthermore the huge difference between the importance of the types of energy in this case study allowed for a simple discrimination in the realization of the standard proposed here. The approach should be further developed in order to extend it for other companies or organizations as suggested by the ADEME - French Environment and Energy Management Agency. In this context it would require to study more general standards involving the different types of energy consumed by organizations.

\section{ACKNOWLEDGMENT}

Presented $\mathrm{PhD}$ work has been developed thanks to the financial support of ADEME (Agence de l'Environnement et de la Maitrise de l'Energie/ French Environment and Energy Management Agency) and APS (Assemblée des Pays de Savoie) and the participation of adixen Vacuum Products.

\section{REFERENCES}

[1] "Win the energy challenge with iso 50001," http://www.iso.org/iso/iso_ 50001_energy.pdf, accessed: 2015-02.

[2] K. Vikhorev, R. Greenough, and N. Brown, "An advanced energy management framework to promote energy awareness," Journal of Cleaner Production, vol. 43, no. 0, pp. 103 - 112, 2013.

[3] R. Kaplan and D. P. Norton, "The balanced scorecard: Measures that drives performance," Harvard Business Review, 1992.

[4] P. Lorino, Méthodes et pratiques de la performance: le guide du pilotage. Les Éditions d'Organisation, 1996.

[5] Qualité et systèmes de management ISO 9000. éditions AFNOR, 2000.

[6] E. Jacquet-Lagreze and J. Siskos, "Preference disaggregation: 20 years of experience," European Journal of Operational Research, vol. 130, no. 2, pp. 233-245, 2001.

[7] G. Bous, P. Fortemps, F. Glineur, and M. Pirlot, "Acuta: A novel method for eliciting additive value functions on the basis of holistic preference statements,' European Journal of Operational Research, vol. 206, no. 2, pp. 435-444, 2010.

[8] "Diviz-decision deck," http://www.decision-deck.org/diviz/, accessed: 2015-03.

[9] Y. Siskos, E. Grigoroudis, and N. F. Matsatsinis, "Uta methods," in Multiple criteria decision analysis: State of the art surveys. Springer, 2005, pp. 297-334. 